\title{
TESTING A SCALED PULSED MODULATOR FOR AN IEC NEUTRON SOURCE INTO A RESISTIVE LOAD*
}

\author{
G.E. Dale ${ }^{\xi}$, R.M. Wheat, Jr., R. Aragonez \\ Los Alamos National Laboratory, P.O. Box 1663, Mail Stop H851 \\ Los Alamos, NM 87545
}

\section{Abstract}

A $1 / 10^{\text {th }}$ scaled prototype pulse modulator for an Inertial Electrostatic Confinement (IEC) neutron source has been designed and tested at Los Alamos National Laboratory (LANL). The scaled prototype modulator is based on a solid-state Marx architecture and has an output voltage of $13 \mathrm{kV}$ and an output current of $10 \mathrm{~A}$. The modulator has a variable pulse width between $50 \mu$ s and 1 ms with $<5 \%$ droop at all pulse widths. The modulator operates with a duty factor up to $5 \%$ and has a maximum pulse repetition frequency of $1 \mathrm{kHz}$.

The use of a solid-state Marx modulator in this application has several potential benefits. These benefits include variable pulse width and amplitude, inherent switch overcurrent and transient overvoltage protection, and increased efficiency over DC supplies used in this application. Several new features were incorporated into this design including inductorless charging, fully snubberless operation, and stage fusing.

The scaled prototype modulator has been tested using a $1 \mathrm{k} \Omega$ resistive load, Test results are given. Short (50 $\mu \mathrm{s})$ and long (1 ms) pulses are demonstrated as well as high duty factor operation $(1 \mathrm{kHz}$ rep rate at a $50 \mu$ s pulse width for a $5 \%$ duty factor). Pulse agility of the modulator is demonstrated through turning the individual Marx stages on and off in sequence producing ramp, pyramid, and reverse pyramid waveforms.

\section{INTRODUCTION}

A pulse modulator for an Inertial Electrostatic Confinement (IEC) neutron source is currently under development at Los Alamos National Laboratory (LANL). The IEC neutron source requires that a high electric potential be maintained between two grids within a hydrogen plasma [1]. In our device, the grids are arranged as concentric spheres, although several other arrangements are also possible. The potential between these grids accelerates the hydrogen ions and creates stable ion orbits. When isotopes of hydrogen, such as deuterium or a mix of deuterium and tritium, are used to form the plasma, neutrons are produced from the nuclear fusion resulting from the energetic collision of these nuclei.

The grid potential, often in the range of $100-200 \mathrm{kV}$, is generally established with DC power supplies. Currentlimiting resistors are used between the power supply and the grid to protect the power supply from overcurrent resulting from an arc within the plasma. While effective at protecting the power supply this current-limiting resistor dissipates a significant amount of power.

The use of a pulsed modulator to supply the grid potential will have several benefits. One is the ability to produce a pulsed source of neutrons from an IEC device. This is important because there are several applications which require a pulsed source of neutrons. The pulsed modulator is also designed to run at a high duty factor, up to $5 \%$. When the modulator is run in this mode the neutron output from the pulsed neutron source is able to approximate a continuous source of neutrons. Therefore, only one power supply is necessary for both pulsed and continuous modes of operation in certain applications.

Another benefit of using a pulsed modulator is that it has the potential to improve system efficiency by removing the current-limiting resistor used to protect the DC power supply. The pulsed modulator proposed for this design has the ability to self limit the current during arcing or shorted load faults. This protection is completely passive yet does not dissipate power during normal operation, increasing the system's efficiency.

The design of the pulsed high-voltage source is based on a solid-state Marx architecture developed at LANL [2]. This paper describes the design, construction, and initial test results of a scaled prototype modulator for an IEC neutron source. The modulator prototype is scaled to $1 / 10^{\text {th }}$ the output voltage of the final design to allow air insulated operation of the prototype.

\section{PULSED MODULATOR DESIGN}

The IEC neutron source under consideration requires a $120 \mathrm{kV}, 10 \mathrm{~A}$ modulator. The pulse width is required to be variable between $50 \mu \mathrm{s}$ and $1 \mathrm{~ms}$ at a duty factor up to $5 \%$, resulting in a pulse repetition frequency ranging from $1 \mathrm{kHz}-50 \mathrm{~Hz}$. The pulse droop must be $<5 \%$ at all pulse lengths.

\footnotetext{
* Work sponsored by Los Alamos National Laboratory under US DOE contract W-7405-ENG-36

$\xi$ email: gedale@lanl.gov
} 
A scaled prototype modulator was constructed to demonstrate the capabilities of the LANL solid-state Marx modulator. The scaled prototype has approximately $1 / 10^{\text {th }}$ the output voltage but the same output current as the fullscale modulator. The characteristics of the demonstration modulator are contained in Table 1.

Table 1. Scaled-prototype modulator specifications.

\begin{tabular}{|c|c|}
\hline Output voltage & $13 \mathrm{kV}$ \\
\hline Output current & $10 \mathrm{~A}$ \\
\hline Voltage droop & $<5 \%$ \\
\hline Peak power & $130 \mathrm{~kW}$ \\
\hline Pulse width range & $50 \mu \mathrm{s}-1 \mathrm{~ms}$ \\
\hline Pulse energy & $130 \mathrm{~J}$ \\
\hline Max duty factor & $5 \%$ \\
\hline Max average power & $6.5 \mathrm{~kW}$ \\
\hline Number of stages & 10 \\
\hline Voltage per stage & $1300 \mathrm{~V}$ \\
\hline
\end{tabular}

The scaled prototype modulator is a 10-stage solid-state Marx bank, the design of which is contained in the references [3].The prototype modulator operates at a stage voltage of $1300 \mathrm{~V} /$ stage for a pulsed output voltage of 13 $\mathrm{kV}$. The LANL solid-state Marx modulator design follows a typical Marx topology except that the charging resistors have been replaced by diodes and the stage switches have been replaced by solid-state switches. In a modification to previous designs a second set of switches are placed in parallel with the upper charging diodes. These switches are open during the output pulse and are closed during the recharge process, giving the stage capacitors a path to ground during charging. The use of these stage-charging switches replaces the charging inductor used in previous designs. This charging inductor would have become prohibitively large for the long pulse lengths required in this application.

A photo of the front of a single modulator board is shown in Figure 1. The stage (lower) and charging (upper) switches and their fiber optic triggering circuitry are shown. The IGBTs are mounted under heat sinks to the left of the center of the board. The two charging diodes are also shown in the lower center part of the board.

A photo of the back of a single modulator board is shown in Figure 2. Each stage contains a single $200 \mu \mathrm{F}$, $1500 \mathrm{~V}$ capacitor [4]. This capacitor provides the energy necessary to achieve $<5 \%$ droop during the long pulse length $(1 \mathrm{~ms})$ operation. A small ferrite transformer located in the center of the board is used to supply isolated utility power to the triggering system. The transformer's primary is a single high voltage wire threaded through the center of all 10 boards and is driven with an $\mathrm{H}$-bridge inverter operating at $100 \mathrm{kHz}$.

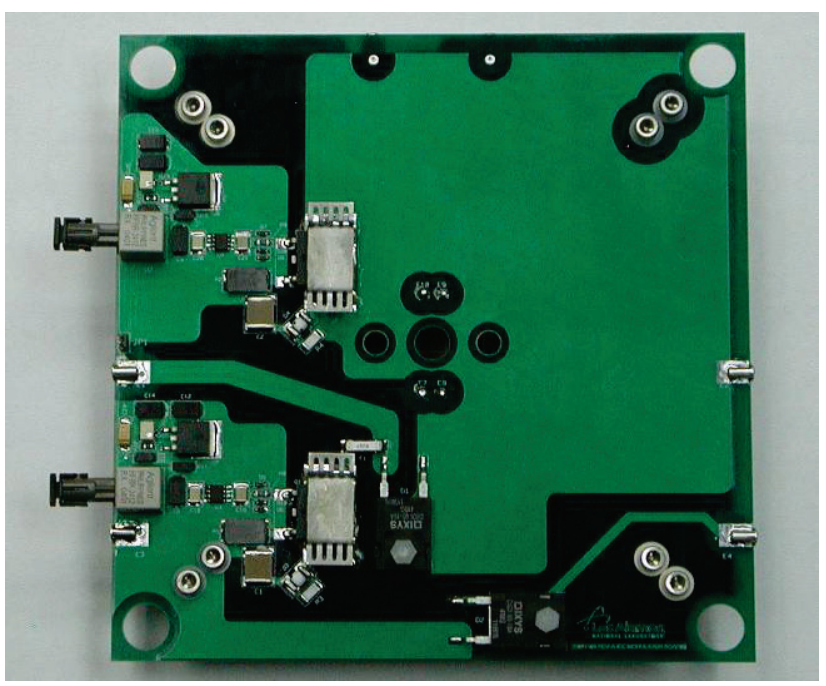

Figure 1. Front view of a single modulator board showing the switching and triggering circuitry. The boards are square and measure $17.8 \mathrm{~cm}$ (7 in) on a side.

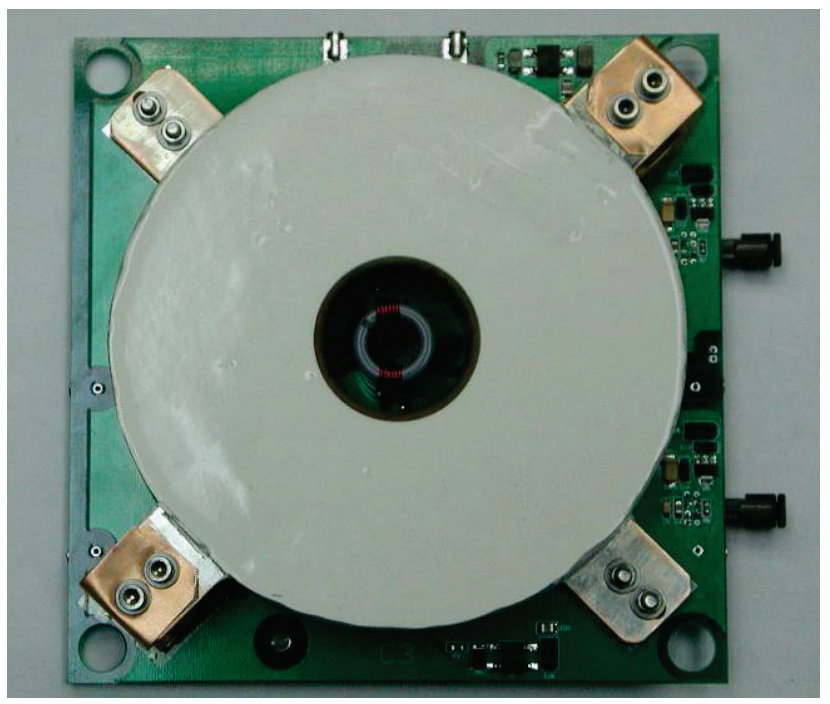

Figure 2. Back view of a single modulator board showing the stage capacitor and utility power system.

A picture of the entire assembly of 10 boards mounted in the modulator chassis is shown in Figure 3. The boards are supported by nylon all-thread rods with nylon spacers between boards. The assembly is mounted to three pairs of nylon feet. The entire assembly is $7 " \times 7 " \times 24$ " long $(17.8 \times 17.8 \times 61.0 \mathrm{~cm})$, not including the height of the mounting feet. The entire modulator is mounted within a modulator chassis which is a 7 unit $(12.25 ", 31.1 \mathrm{~cm})$ high 19 " rack mountable chassis measuring 26 " $(66 \mathrm{~cm})$ deep with 16.37" $(41.6 \mathrm{~cm})$ interior width. Also contained in the modulator chassis are the fiber-optic trigger system and the auxiliary power supply for the modulator stages. 


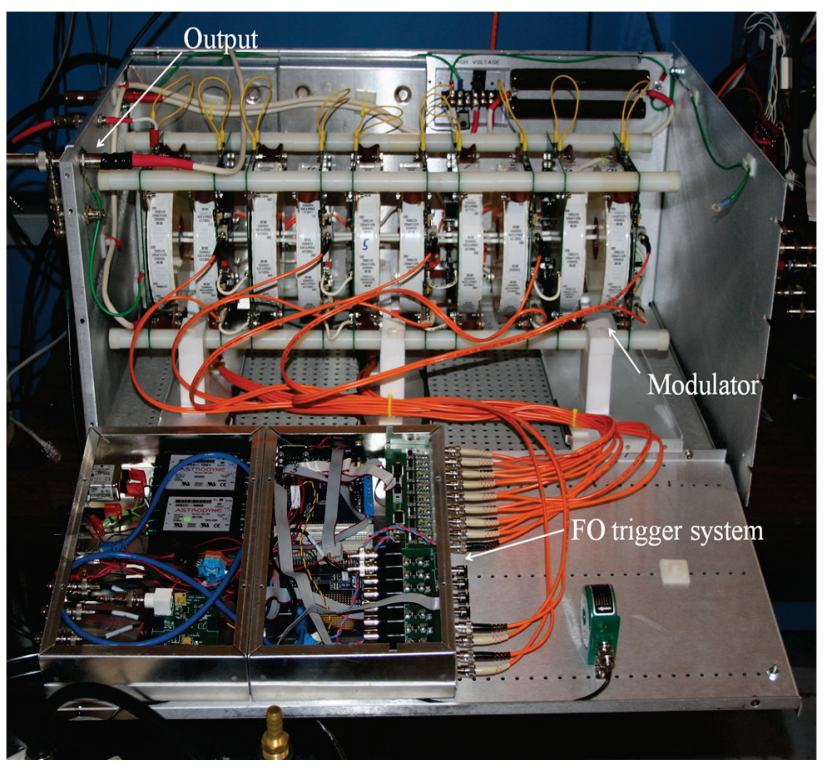

Figure 3. Picture of the Marx assembly shown mounted in the modulator chassis. Also shown are the fiber-optic (FO) trigger system and the auxiliary power supply.

The fiber-optic trigger system is based on a 2 microcontroller design developed at LANL [5]. One microcontroller controls all triggering and timing functions. The output of the trigger and timing microcontroller is sent to several fiber-optic transmitter boards which send the trigger signals over fiber-optic cables to fiber-optic receivers located on each modulator stage. The second microcontroller controls all communications between the user and the trigger and timing microcontroller. In this implementation, Ethernet was used to communicate between the user's computer and the communications microcontroller. The 2 microcontrollers communicate between themselves using a serial interface protocol. The functions of communications and trigger timing are split between 2 microcontrollers for a better distribution of resources and to optimize the individual microcontroller clock frequencies for the specified application.

The auxiliary power supply consists of an H-bridge inverter driving a single turn primary used to supply isolated power to each stage of the modulator. The inverter operates at $100 \mathrm{kHz}$ and $5 \mathrm{~V}$ output per stage, or $50 \mathrm{~V}$ output for the 10 stage modulator used here. Each stage of the modulator has a small transformer consisting of a 6 turn secondary wound around a ferrite toroid through which the single turn primary from the auxiliary power supply is threaded. With a 6:1 transformer ratio the secondary auxiliary voltage is $30 \mathrm{~V}$ with a $5 \mathrm{~V}$ drop per stage core on the primary. This secondary power is rectified and regulated to provide the gate voltage (adjustable between 12 and $20 \mathrm{~V}$ ) and $5 \mathrm{~V}$ for the fiberoptic receiver electronics.

\section{DESIGN FEATURES}

The use of all solid-state components in a Marx architecture provides many benefits. These benefits are:

Variable pulse length: The switches for each stage are controlled via fiber-optic control signals, allowing independent control of the pulse width of each stage.

Variable amplitude: The ability to independently control the pulse width of each stage provides the capability to rapidly vary the output amplitude, which can be used to create complex output waveforms. When used to power an IEC neutron source the variable amplitude capability can be used for rapid adjustment of the neutron intensity.

Variable pulse repetition frequency: The fiber-opticstage triggering allows the pulse repetition frequency to be adjusted on a pulse to pulse basis. The modulator can also be operated in burst mode, for a train of several pulses spaced very close together.

Transient switch over-voltage protection: The modulator has the inherent ability to protect the switches from voltage transients caused by inductive loads. We refer to this capability as self snubbering because it resembles the protection of a snubber but without the extra components or the power dissipation of a traditional snubber [6].

Over-current protection: The modulator uses emitter feedback to limit the switch current during an arc or a shorted load fault. This protection is passive, requiring no rapid intervention by the user or the control system. It is also efficient because the dissipative protection is active only when there is an over-current condition.

The design of the scaled prototype also includes several enhanced features. These enhanced features are:

Long pulse length: The pulse length of this modulator is up to $1 \mathrm{~ms}$ with $<5 \%$ droop. Previous modulators of this design had pulse lengths on the order of $5 \mu \mathrm{s}$. This long pulse length with low droop was achieved by using $200 \mu \mathrm{F}$ high-energy-density stage capacitors. There are 10 of these capacitors in the prototype modulator, each of which stores $169 \mathrm{~J}$ at a charge voltage of $1300 \mathrm{~V}$ per stage.

Inductorless charging: Previous modulators of this design used a charging inductor to complete the pathway between the stage capacitors and ground during charging. The long pulse length required of this modulator precludes the use of a charging inductor, since its size would be prohibitively large. For this design we used active switching to complete the charging pathway. The switches are controlled with fiber-optic trigger signals and are only on when the load switches are open.

Snubberless operation: This concept was explored in previous designs but this is the first design to fully implement the concept. Snubbers are generally designed for a given pulse width and load inductance. Being able to operate without snubbers improves this design's pulsewidth variability and pulse repetition variability without 
limiting the protection given to the switches. Traditional snubbers also dissipate power and so snubberless operation improves efficiency. Since snubberless operation offers full switch protection independent of load inductance, the modulator can fire into almost any inductance without the worry of switch failure.

Stage fusing: A stage-isolating fuse was incorporated into this design. If a switch fails short the high current through the fuse will cause it to open. This will fully isolate the stage with the failed switch. The output from the remaining stages bypasses the isolated stage through the charging diodes.

High duty factor: Previous designs of this type were operated at a low duty factor of $\sim 0.06 \%$. This modulator is designed to operate at a significantly higher duty factor of up to $5 \%$. The maximum duty factor of this design is limited only by the output current of the capacitorcharging power supply. The duty factor could be increased further by using a higher current capacitor charging supply.

\section{RESULTS}

The following figures contain preliminary test results from the scaled prototype modulator. These test results are for a stack of 10 boards fired into a $1 \mathrm{k} \Omega$ load. The boards are protected against a short circuit fault by emitter feedback and the fault current under these conditions is limited to $28 \mathrm{~A}$. The boards are completely snubberless in these tests.

The short pulse $(50 \mu \mathrm{s})$ capability of the modulator is shown in Figure 4. This data is for a 10-stage stack fired into a $1 \mathrm{k} \Omega$ resistive load. The charge voltage is $1000 \mathrm{~V}$ per stage for an output voltage of $10 \mathrm{kV}$. The output current is 13 A. Droop is negligible. Even shorter pulse lengths are possible with the modulator, limited only by the rise and fall time of the switches.

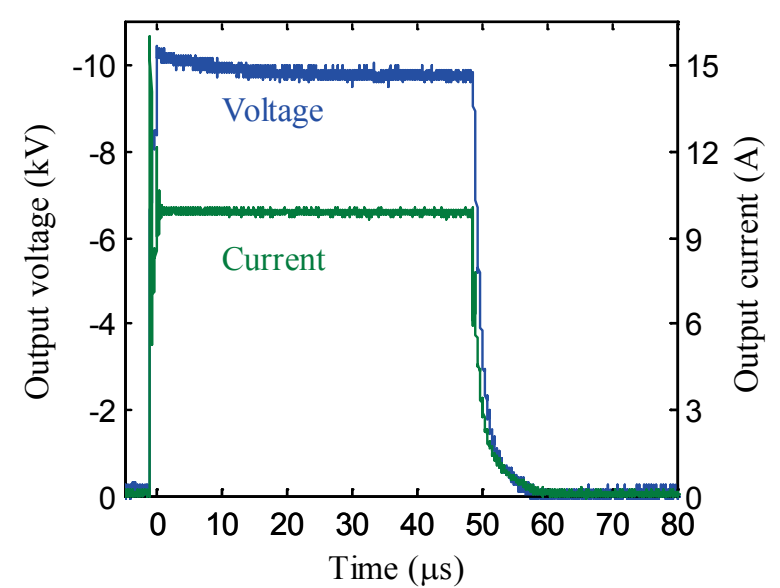

Figure 4. Short pulse $(50 \mu \mathrm{s})$ operation of a 10-stage stack fired into a $1 \mathrm{k} \Omega$ load.

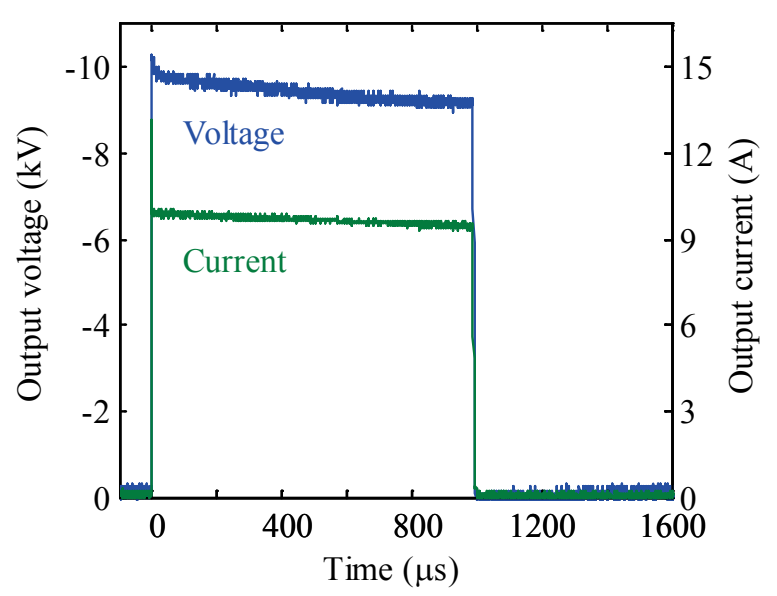

Figure 5. Long pulse (1 ms) operation of a 10-stage stack fired into a $1 \mathrm{k} \Omega$ load.

The long pulse $(1 \mathrm{~ms})$ capability of the modulator is shown in Figure 5. This data is for a 10-stage stack fired into a $1 \mathrm{k} \Omega$ resistive load. The charge voltage is $1000 \mathrm{~V}$ per stage for an output voltage of $10 \mathrm{kV}$. The peak output current is $10 \mathrm{~A}$ and the droop is $\sim 5 \%$.

The high duty factor capability of the modulator is shown in Figure 6. This figure contains the output voltage waveform for a $50 \mu$ s pulse operated at $1 \mathrm{kHz}$, which is a $5 \%$ duty factor. Four output pulses are shown.

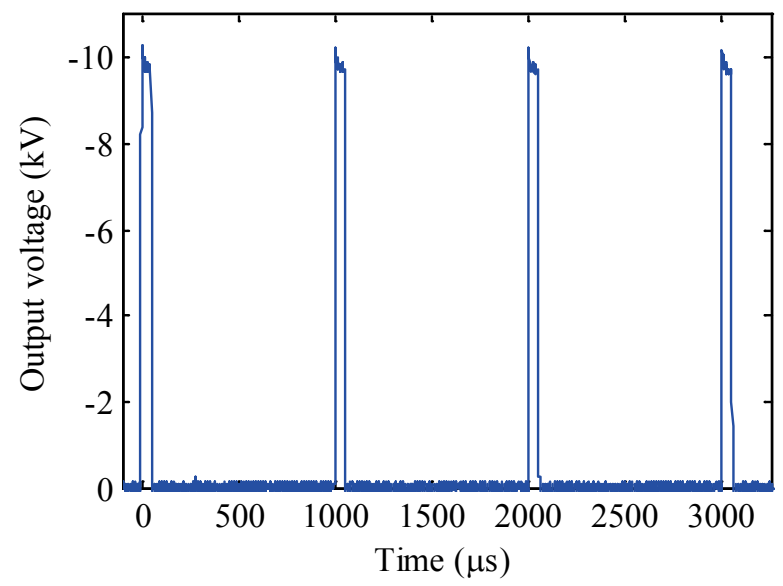

Figure 6. High duty factor operation of a 10-stage stack fired into a $1 \mathrm{k} \Omega$ load. The pulse width is $50 \mu \mathrm{s}$ and the device is operating at $1 \mathrm{kHz}$ for a $5 \%$ duty factor, 4 pulses are shown.

The diode charging rails of the modulator allows it to fire stages individually. Stage switches that are not closed are simply bypassed by the output current through the diode charging rail. This capability allows the modulator to produce highly adjustable waveforms. This is demonstrated in the next three figures. In all figures the modulator was fired into a $1 \mathrm{k} \Omega$ load with a charge voltage of $1000 \mathrm{~V}$ per stage.

Figure 7 shows a 10 step ramp waveform created by sequentially turning on stages of the modulator. Figure 8 
shows a 10 step pyramid created by sequentially turning the 10 stages of the modulator on and then off. Figure 9 shows a 10 step inverted pyramid created by turning all the modulator stages on, then sequentially turning sages off and then on again.

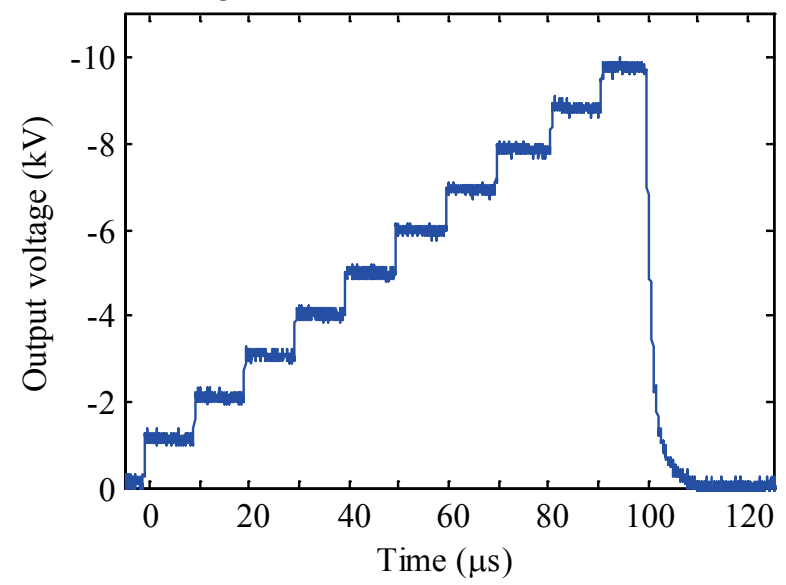

Figure 7. Ramp waveform created by turning the 10 stages of the modulator on in sequence.

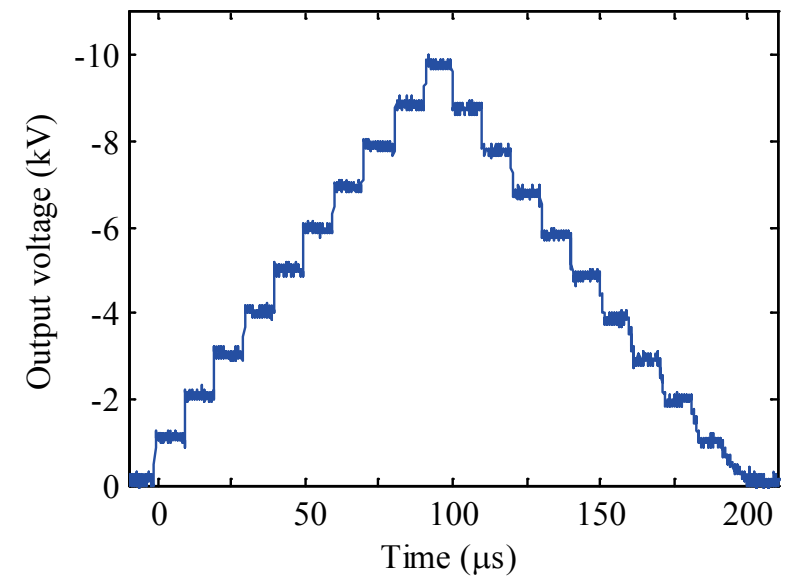

Figure 8. Pyramid waveform created by sequentially turning the 10 stages of the modulator on and then off

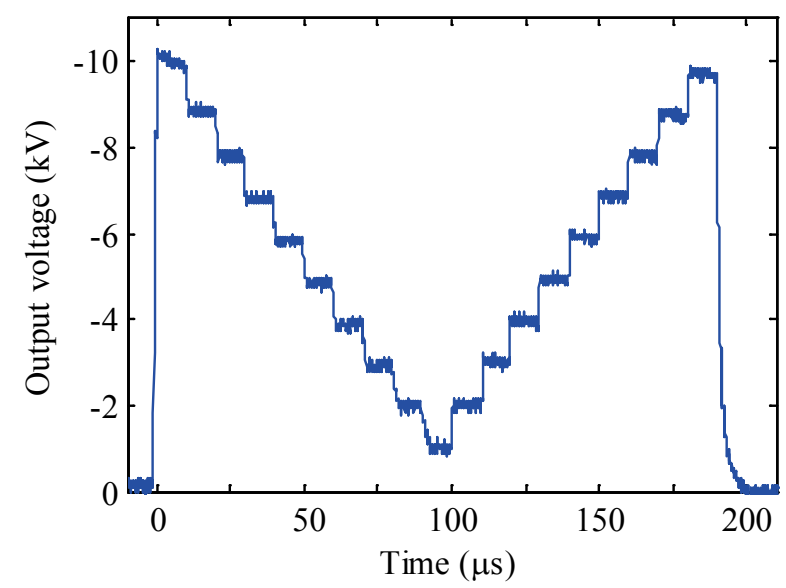

Figure 9. Inverted pyramid waveform created by turning all the modulator stages on, then sequentially turning sages off and then on again.

\section{CONCLUSIONS}

A scaled prototype modulator for an IEC neutron source was designed and constructed at LANL. The design incorporates a solid-state Marx architecture with actively switched charging. Using a pulsed solid-state Marx modulator to drive an IEC modulator will allow the neutron source to have multiple enhanced output capabilities such as single pulse, high-frequency burst, and continuous pulsed modes. The output amplitude and pulse width will also be adjustable on a pulse to pulse basis. The modulator design also offers several reliability enhancing features such as transient switch over-voltage protection, switch over-current protection, and stage fusing.

Preliminary test results for a 10-stage stack fired into a resistive load were shown. All tests were performed without traditional snubbers protecting the switches. High duty factor operation was demonstrated by running the modulator with a $50 \mu$ s pulse width at a $1 \mathrm{kHz}$ rep rate for a duty factor of 5\%. Waveform control was demonstrated through the creation of ramp, pyramid, and inverting pyramid waveforms.

\section{REFERENCES}

[1] J.F. Santarius, et al., "Overview of University of Wisconsin Inertial-Electrostatic Confinement Fusion Research," Fusion Science and Technology, Vol. 47 (2005).

[2] G.E. Dale, et al., "Design and Application of a DiodeDirected Solid-State Marx-Modulator," in Proc. 15th IEEE International Pulsed Power Conference, Monterey, CA (2005).

[3] G.E. Dale, "Pulsed Modulator for an IEC Neutron Source," in Proc. 16 ${ }^{\text {th }}$ IEEE International Pulsed Power Conference, Albuquerque, NM (2007).

[4] T.A. Hosking and M.A. Brubaker, "Annular form factor film capacitors," in Proc. 15th IEEE International Pulsed Power Conference, Monterey, CA (2005).

[5] R.M. Wheat Jr. et al., "Multiple Output Timing and Trigger Generator," Proc. $17^{\text {th }}$ IEEE International Pulsed Power Conference, Washington, DC (2009).

[6] G.E. Dale and H.C. Kirbie, "Power Flow Management in a Diode-Directed Solid-State Marx Modulator," in Proc. $27^{\text {th }}$ IEEE International Power Modulator Symposium, Washington D.C. (2006). 